\title{
New forms of pharmaceutical co-crystals of gefitinib with resorcinol \\ Parimaladevi Palanisamy
}

Department of Chemical Sciences, University of Limerick, Ireland, V94 T9PX

p.palanisamy@ul.ie

Gefitinib is a chemotherapeutic drug used in the treatment of breast and lung cancer. It is belonging to Biopharmaceutical Classification System II (BCS II) as it is highly permeable and poor soluble drug. The bioavailability of gefitinib is significantly affected by low aqueous solubility of the drug. To overcome this issue, we prepared co-crystals of gefitinib with resorcinol using dry grinding and solution growth method. Crystal structures of newly synthesized co-crystals were analysed by single crystal X-ray diffractometer, and it reveals that gefitinib formed 1:1:1 co-crystal with resorcinol and water. Quantitative analysis intermolecular interactions were studied using Hirschfeld surface and 2D fingerprint plot analysis. Weak molecular interactions like $\mathrm{H}^{\cdots} \mathrm{C}, \mathrm{H} \cdots \mathrm{O}$, $\mathrm{Cl} \cdots \mathrm{H}, \mathrm{F} \cdots \mathrm{H}$ and $\mathrm{N} \cdots \mathrm{H}$ interactions play a significant role in gefitinib co-crystal formation.

Keywords: Co-crystals, gefitinib, resorcinol 